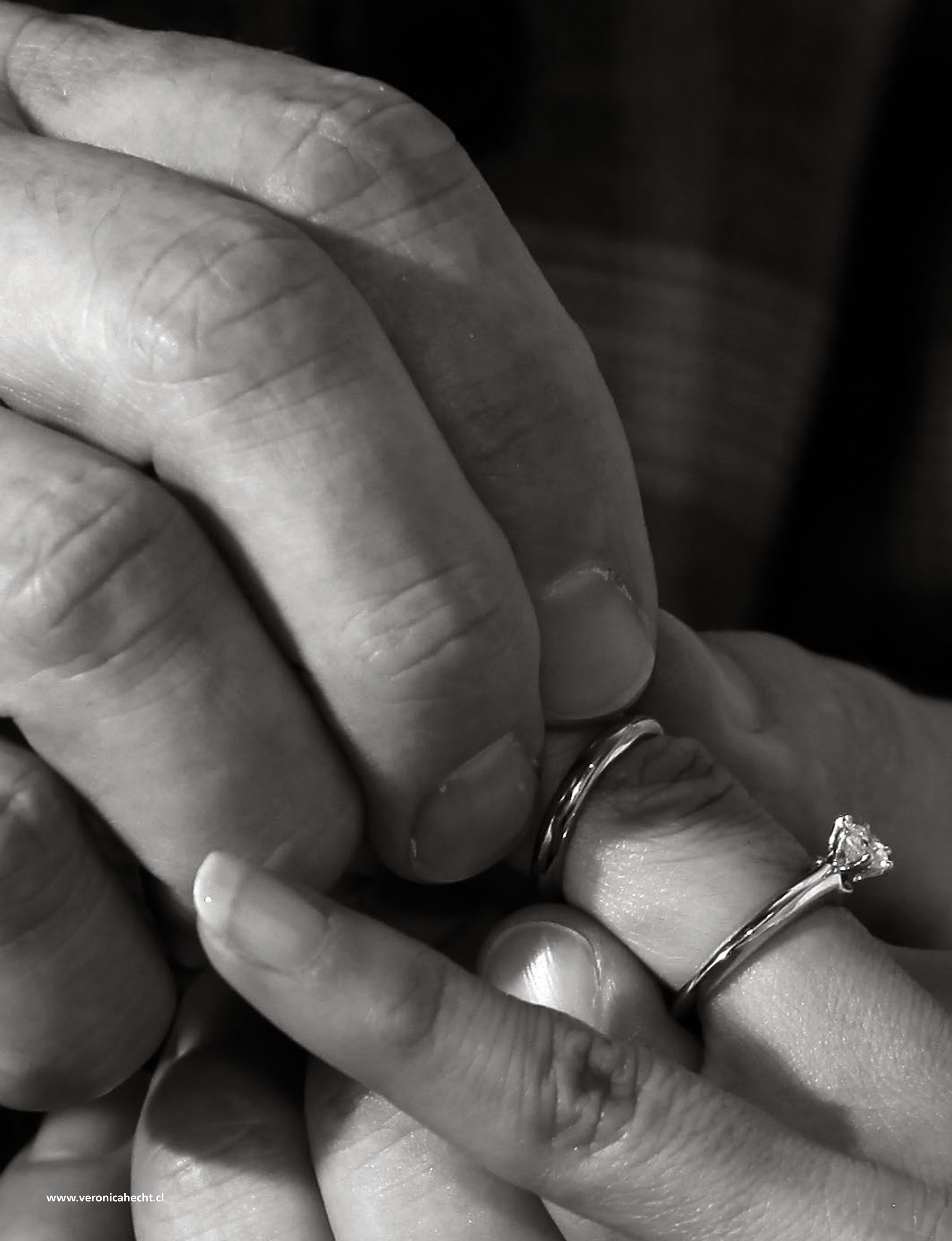




\section{Desentrañando los sentidos del anillo de matrimonio}

Víctor Alfonso Nieto

En este trabajo se presenta el resultado de una exploración del valor simbólico del anillo de matrimonio en el contexto contemporáneo de la Ciudad de México, con base en la triple relación usuarioobjeto-discurso; el caso se centra en mujeres usuarias. ¿Qué significa el anillo de matrimonio desde el punto de vista institucional?, ¿cómo transforman los usuarios, más concretamente las mujeres, dicho significado?, ¿con qué elementos contextuales se relacionan los posibles sentidos de la argolla? Son las interrogantes que se abordarán en este texto, en el cual se presenta el resultado de una pesquisa centrada en usos normativos y no normativos del anillo de matrimonio.

Palabras clave: anillo de matrimonio, uso normativo, valor simbólico 


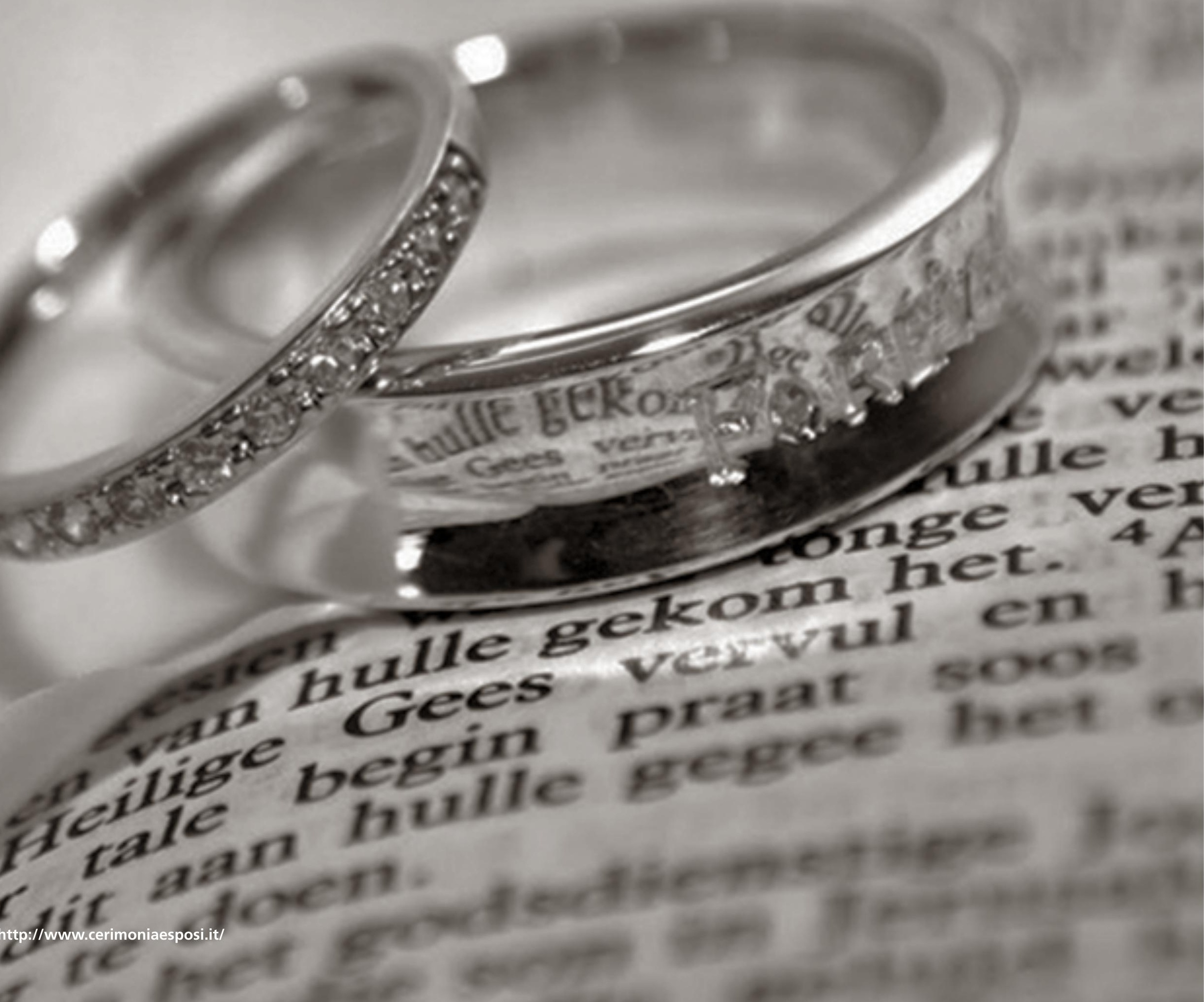


Quizás sean los egipcios los usuarios más remotos de los anillos en general, para quienes la forma circular este objeto representaba la eternidad y la unidad (Robb, 2011). Sobre el uso del anillo como símbolo de una promesa, identificamos antecedentes en la Roma antigua, donde el novio enviaba a su futura esposa un anillo de hierro para expresar su voluntad de mantener una relación duradera.

No hay conclusiones definitivas al respecto de por qué este objeto se porta en el dedo anular, si bien existe cierto consenso en relación a que éste se conecta directamente con el corazón mediante una vena, razón simbólica que justifica la ubicación de la alianza matrimonial en ese lugar del cuerpo (Monger, 2004, p. 234).

Para la Edad media, el intercambio de anillos de matrimonio con inscripciones en su interior era una práctica bien establecida:

A popular form of betrothal ring was the gimmal ring, two or three interlocking rings that joined together to form one complete ring. Many of these had a central motif of two hands clasped over a heart. The ring would be solemnly broken over the Bible, before witnesses, at the bethrotal, each of the couple receiving a part of the ring (p. 234).

Paul Berdanier (1939) señala que la obligación del uso del anillo para las ceremonias de petición de mano deriva de una antigua práctica en la cual el varón ataba un cordón alrededor de la cintura, caderas y tobillos de la mujer de la que estaba enamorado, para asegurarse que su espíritu se encontraba bajo su control. Esta noción de vínculo estrecho con el otro -que en el caso citado connota posesión pero que en otros contextos puede entenderse en sentidos más amplios, simplemente como un lazo íntimo entre cónyuges - se mantiene hasta nuestros días, extendiéndose al caso de los matrimonios entre personas del mismo sexo (Ayers y Brown, p. 133).

El uso del anillo matrimonial se convirtió en una práctica ampliamente extendida hasta el siglo XIX y el uso de la alianza en ambos cónyuges se popularizó a mediados del siglo XX. De acuerdo con Vicki Howard (2003), la inclusión de dos anillos data de 1944 y se relaciona estrechamente con la coyuntura de la industria joyera durante la posguerra y con la transformación de las creencias en torno al género y al compromiso, sobre todo en lo que hace a la clase media estadounidense.

Muchas creencias —religiosas y no religiosas — se asocian con el anillo de matrimonio, cuyo metal precioso simboliza la pureza y durabilidad del amor. Los posibles significados de este objeto también incluyen supersticiones que relacionan el destino del anillo con el destino de la pareja que lo porta, de tal suerte que en algunos contextos culturales se considera como mal augurio su pérdida, incluso su caída antes de la boda; en algunas culturas también se les confieren propiedades curativas o mágicas (Monger, p. 235). En lo que hace a la religión, ejemplos como The Golden Wedding Ring or Observations on the Institution of Marriage (Clowes, 1832) y la obra de teatro El taller del orfebre (Wojtyla, K. 2013) dan cuenta del significado de este objeto:

Here we both are, we grow out of so many strange moments, as if from the depths of facts, ordinary and simple though they are. Here we are together. We are secretly growing into one because of these two rings (Wojtyła, K. J. 1960 p.8).

Más allá de su potencial real para unir, curar o generar desgracias, es un hecho que el anillo de matrimonio es un poderoso portador de sentidos con valor instrumental, es decir, estrechamente vinculados con el comportamiento. La argolla refiere en primera instancia al hecho de que quien lo porta sostiene una relación exclusiva -emocional, social, sexualmente- con alguien más, con la expectativa convencional de mantenerla así.

En este orden de ideas, estudios como "Human Mate Choice and the Wedding Effect. Are Married Men More Atractive?" de Uller y Johansson (2003), exploran la conducta colectiva en relación a este significado, planteándose si las mujeres experimentan un mayor interés en los varones heterosexuales que portan un anillo de matrimonio. Por su parte Shirley Ogletree (2010) se pregunta en "With this Ring, I Thee Wed: Relating Gender Roles and Love Styles to Attitudes towards Engagement Rings and Weddings" acerca de las diferentes aproximaciones que hombres y mujeres tienen al respecto de las argollas y la boda, encontrando reveladoras diferencias de género, por ejemplo en lo que hace a la conservación del anillo por parte de las mujeres aún cuando la relación conyugal se haya roto. Este tipo de aproximaciones dan cuenta de la gran relevancia que el sentido de este objeto y su relación con la experiencia tiene en nuestros días, sentido que abreva de tradiciones remotas pero que también añade nuevos significados relacionados con el mundo contemporáneo que habitan sus usuarios y usuarias.

En el contexto de México, la cultura refiere claramente a los significados y comportamientos asociadas con el sentido tradicional de este objeto tal como se hace patente en los fragmentos de estas canciones populares. 


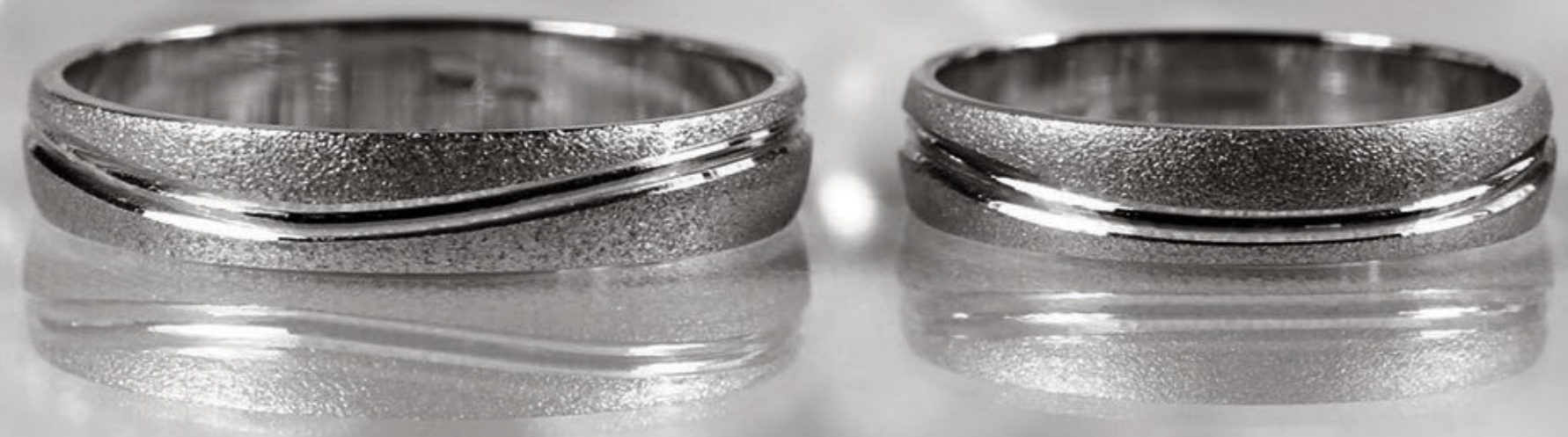

\section{Anillo de compromiso}

Cuco Sánchez (Sánchez Saldaña, 1994):

Anillo de bodas que puse en tus manos, anillo que es símbolo de nuestro amor, que unió para siempre y por toda la vida, a nuestras dos almas delante de Dios.

\section{Anillo grabado}

Antonio Aguilar (1991):

Toma este anillo

que lleva mi nombre grabado

tómalo y póntelo

en el lado del corazón.

\section{Par de anillos}

Grupo Viento y Sol (1991):

Te prometo que el domingo Haré realidad tu sueño

Entraremos a la iglesia

Para ser al fin tu dueño

Y cuando demos el sí

Lo haremos con la esperanza

De no quitarnos jamás

Amor este par de alianzas

Este par de anillos

Con nuestros nombres grabados

Este par de anillos

Para dos enamorados 
En suma, los anillos de matrimonio hablan. Nos hablan de rituales y creencias relativas al amor, la exclusividad sexual y el sentido de pertenencia. El sentido y el uso del anillo están prescritos, es decir, existen un conjunto de acuerdos más o menos estables acerca de la ocasión, el uso, el material, el discurso y el sentido asociados con este objeto. Pero, ¿qué tan flexibles son estas prescripciones? ¿cómo transforman estos acuerdos quienes usan el objeto? ¿qué piensan y hacen las mujeres que poseen una argolla matrimonial en relación al discurso tradicional, por cierto muy potente? Al respecto de esto último, Parsons (2008) señala:

Despite the efforts of our feminist foremothers to create various models of feminine achievement, one traditional mandate for hetero-feminine success still reigns supreme: find the right man, get married, and settle down with a family. The wedding ring often functions as a symbol of accomplishment in this model, and while heterosexuals may don rings with mere intent to make public their commitment, the ring tends to communicate much more than this in practice. In a heterosexist context it indicates membership in a privileged group - the group of married heterosexuals - and it can indicate a kind of social achievement, or even specific desirable attributes, such as maturity or stability. While a ring communicates these things about both heterosexual men and women, a ring on the finger of a heterosexual woman can additionally communicate that she is "fulfilled," that she is "taken," or that she has reason to be content.10 It can indicate that she has someone (a man) who loves her and has committed to her -an achievement that is often considered of highest importance, and that can still trump having a good career or close friends (p. 396).

\section{Discusión}

Para realizar este estudio se llevó a cabo una investigación documental y se registraron 4 casos específicos de usuarias entre 20 y 45 años, residentes en la Ciudad de México, con el propósito de entender las diversas formas en que puede manifestarse la triple relación entre sujeto, objeto y discurso; la selección de los casos siguió un sistema flexible sin propósitos exhaustivos, similar al indicado para la conformación de grupos focales (Merton, 1990): se eligieron a informantes que poseyeran un anillo de matrimonio con independencia de su estado civil y sin que la posesión del objeto implicara utilizarlo en el dedo anular de la mano izquierda. Las entrevistadas no se conocía entre sí, correspondían a los estratos socioeconómicos C, C+ y A/B, (Mc Daniel y Gates, 2005) no recibieron una remuneración económica por su testimonio y fueron informadas acerca de los propósitos del estudio. Para la lectura de los resultados deberá considerarse este sesgo metodológico, que responde al enfoque cualitativo de la investigación.
Las entrevistas se realizaron en los meses de abril y mayo del 2013. Mediante un cuestionario semiestructurado se exploró el sentido del anillo matrimonial; puede sonar contradictorio el hecho de llamar al objeto de ese modo aun en el caso de las mujeres solteras, sin embargo las entrevistas corroboraron que todas las informantes reconocían el objeto como tal en primera instancia, si bien los posibles usos pueden alejarse de la prescripción social, esto es, utilizar la argolla de matrimonio como resultado de un ritual de unión con fines conyugales.

La entrevista se diseñó con el propósito de explorar la relación entre el objeto, lo que la usuaria dice acerca de él y el sentido que le confiere, con énfasis en posibles significados que tomen distancia del sentido tradicional; el instrumento consistió en siete preguntas cuya calificación se presenta en la tabla 1. Se citan textualmente las respuestas más relevantes para los fines de este estudio.

Tras analizar las entrevistas se concluyó lo siguiente:

\section{Se identificaron usos no normativos del objeto que abrevan del sentido tradicional.}

Debido a la configuración del corpus, fue sencillo identificar usos aparentemente no prescriptivos del anillo matrimonial. Dos de las informantes poseen una argolla sin estar casadas y si bien reconocen el sentido de "unión conyugal", este valor es transformado para referir a la unión madre-hija y a la amistad entre tres mujeres heterosexuales. Sin duda, este uso hace referencia a una comprensión más flexible del sentido del matrimonio como unión exclusiva, estrecha y profunda con otra(s) persona(s), sin que ello conlleve el intercambio sexual. En este sentido se detecta una ampliación del sentido del concepto matrimonio o bien una reducción al valor de "unión emotiva profunda con la Otredad" como atributo mínimo para que la argolla opere simbólicamente como tal.

www.tiffany.com.mx

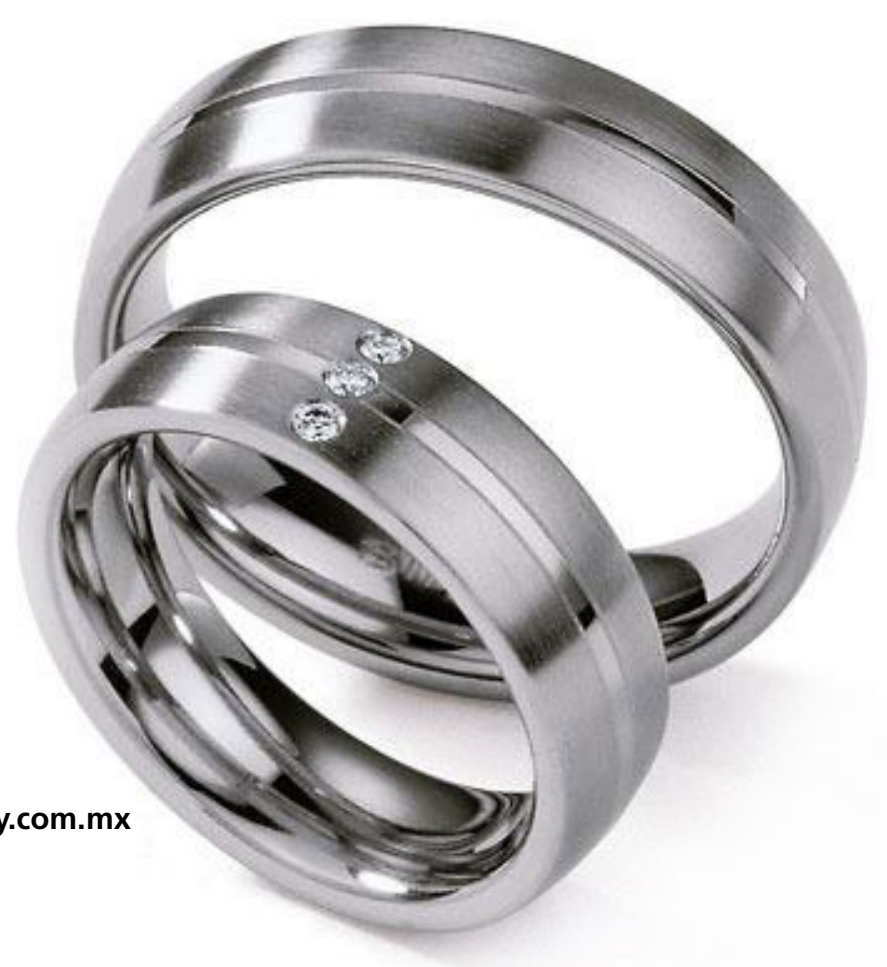


2. Existe una relación entre el material, el uso y el sentido de la argolla

La observación de los anillos y el reactivo en relación a la argoIla perfecta permitieron detectar una estrecha relación entre el material con el cual se elabora el objeto y su significado. Las dos informantes que no están casadas poseen anillos de plata y no de oro blanco o amarillo; las cuatro informantes refirieron a los materiales, tres de ellas consideraron que el anillo perfecto debería ser de oro; tres enfatizaron en el ritual asociado con la recepción del anillo (boda o aniversario de bodas), dando cuenta de la preeminencia del valor tradicional que vincula al objeto con la unión conyugal, siguiendo a Parsons (2008). Resulta de especial interés el hecho de que todas las informantes hicieron referencia a la sencillez de la pieza como un valor de la argolla ideal: el anillo de bodas perfecto es de oro, pero no incluye piedras preciosas excesivas, lo cual indica una relación entre el diseño y el valor del objeto que convendría explorar en estudios subsiguientes.

En lo que refiere a los rituales, las entrevistas arrojan resultados coincidentes con las reflexiones que la antropóloga Lucy Mair (1971) formulase a principios del siglo XX en relación al intercambio de símbolos como componente neurálgico para la validación del matrimonio; en este orden de ideas, el intercambio de argollas en aquellas culturas donde la práctica anida, constituye un momento crucial en el paso de los contrayentes hacia el ámbito de las personas casadas y en este sentido, el objeto se torna en un verdadero símbolo del nuevo estatus adquirido.

3. El aparente uso no normativo del anillo no modifica el valor de "unión emotiva profunda"

Sólo una de las usuarias utiliza el anillo en el dedo corazón de manera permanente. Otra de ellas no lo lleva en la mano, una más lo utiliza sólo cuando va a viajar o para salir con sus amigas, la última lo utiliza en el dedo medio. Todas reconocen el valor del anillo como símbolo de la unión con alguien más y este sentido no se modifica por el hecho de que la argolla se porte en otro dedo o no se porte.

Al respecto de la no portación del anillo, en los dos casos registrados existen motivos poderosamente vinculados con el Otro: una de las informantes no utiliza su anillo porque su cónyuge tampoco lo hace, otra de las entrevistadas no lo usa aludiendo a que le estorba, si bien más adelante hace referencia a que su cónyuge extravió la argolla. Por lo tanto, incluso cuando el anillo no se porta, hace relación al estatus que guarda la usuaria con respecto a una otredad cuyo comportamiento está prescrito y ejerce consecuencias sobre el uso del objeto.

\section{El discurso en torno a la argolla hace referencia a creen- cias tradicionales y no tradicionales}

Las cuatro informantes reconocieron en la argolla el valor del matrimonio (civil y/o religioso), aun cuando no estén casadas o no planeen estarlo. Todas reconocieron la exclusividad emotiva -no necesariamente sexual- como principal contenido asociado con el objeto; el dicho de las informantes hace referencia a la otredad, que abarca una extensa variedad incluyendo el cónyuge, los familiares, los amigos, los desconocidos en los diversos espacios de convivencia, entre otros: esto es, la argolla parece referir en primera instancia a la relación Alter/Ego, pero no exclusivamente, puesto que las usuarias reconocen como actores sociales referidos en su contenido a otros tantos que de alguna manera contribuyen a dotar al objeto de sentido.

En todos los casos el discurso dio cuenta de un valor sentimental asociado con el objeto, que puede ser o no sustituible (no así la emoción asociada), si bien la pérdida de la argolla significaría un hecho lamentable. Este valor reproduce el contenido "unión exclusiva profunda" tradicional, pero toma distancia del contenido "unión conyugal", dependiendo de las características y el estilo de vida de la usuaria. En coincidencia con Ogletree (2010), las cuatro usuarias confirmaron que guardarían el anillo - acción que se supone, preservaría el valor simbólico del objeto- en caso de que no pudieran llevarlo consigo: este hallazgo nos remite directamente a la preservación del contenido simbólico del objeto en los términos que Marcel Mauss formulase al respecto de los dones en su célebre ensayo The Gift (Mauss, 1967).

Muchas tareas pendientes quedan en el tintero. Esta exploración señala la pertinencia de excavar más profundo en el imaginario colectivo, quizás mediante entrevistas a una selección más amplia de informantes, incluyendo a hombres y mujeres heterosexuales y homosexuales, casados o no, con el propósito de identificar un repertorio más amplio de vínculos y sentidos asociados con este objeto. 
Caso 1

Características del objeto

¿Cómo y cuándo llegó

este anillo a tu vida?

¿En qué situación lo usas?

¿Qué significa

este anillo para ti?

¿Qué crees que significa este anillo para las demás personas?

¿Qué pasaría si este anillo se extraviara? ¿Cómo te sentirías? ¿Qué harias?

Si en algún momento de tu vida, tuvieras que dejar de usarlo, ¿Qué harías con él?

Si pudieras tener el anillo perfecto, ¿cómo sería? ¿en qué circunstancias habria llegado a tu vida?
Mujer con 41 años de edad, heterosexual, católica, casada, madre de dos hijas, profesora-investigadora.

Oro blanco con bajorrelieve, grueso, con fecha de matrimonio y nombre del cónyuge grabado en el interior, moleteado.

Matrimonio, hace 12 años.

Oro blanco con bajorrelieve, grueso, con fecha de matrimonio y nombre del cónyuge grabado en el interior, moleteado.

"Respeto, la unión que va de la mano con un respeto, un compromiso, amor, honestidad, lealtad"

"Para algunos hombres, el hecho de que no traigas anillo puede significar que eres una mujer que no tienes compromiso o ataduras, es decir que eres una mujer soltera; para algunos otros que no lo portes, también puede significar esta parte de que te estorba el mismo anillo por tus actividades laborales; (...) están también las que pueden opinar que ya no te casaste o que ya te separaste $y$, finalmente, para otras que puede que no lo usen (...), bueno como no quieres que te vean que tienes un compromiso, pues no lo usas."

Lo lamentaría por el valor sentimental pero sería una oportunidad para elegir otro de su gusto.

Actualmente ha dejado de usarlo cotidianamente dado que su marido dejó de usarlo, de manera que está guardado.

"Mi anillo de matrimonio perfecto tendría que ser uno que trajera unos diamantes, chiquitos, algo así muy discreto. Y sobre el momento en que llegará a mí, no sería uno, tendrían que ser dos. El primero, que ya yo en una edad más madura y con la posibilidad económica de adquirirlo me dijera: 'esto es lo que siempre soñé, me lo merezco' y lo comprara. Mientras que la segunda es que me encontrara a mi alma gemela, que tuviera las posibilidades de comprarme un anillo como esos y entonces le diría: 'este es', pero a lo mejor no tendría que decirle porque como sería mi alma gemela, entonces sabría que ese es." 


\section{Caso 2}

Características del objeto

¿Cómo y cuándo llegó

este anillo a tu vida?

\section{¿En qué situación}

lo usas?

¿Qué significa

este anillo para ti?

¿Qué crees que significa este anillo para las demás personas?

\section{¿Qué pasaría si este}

anillo se extraviara?

¿Cómo te sentirías?

¿Qué harías?

\section{Si en algún momento}

de tu vida, tuvieras

que dejar de usarlo,

¿Qué harías con él?

\section{Si pudieras tener}

el anillo perfecto, ¿cómo sería? ¿en qué circunstancias habria llegado a tu vida?
Mujer con 20 años de edad, heterosexual, católica, soltera, estudiante de nivel superior.

Argolla de plata con bajorrelieve, inscripción de fecha y nombre del cónyuge en la parte interior, con bordes rebajados.

Encontró los anillos de sus padres haciendo limpieza profunda. Se los devolvió y su madre no volvió a usar el suyo. Lo encontró hace tres años.

De manera cotidiana, en el dedo medio de la mano derecha.

"Significa que traigo algo de mi mamá conmigo todo el tiempo: en las pocas ocasiones en que tengo que quitármelo siento que es tiempo al que tengo que renunciar a su recuerdo, ya comente la distancia que nos mantiene separadas y es que a mi mamá la quiero mucho. Para mí es traer algo conmigo que me recuerda a mi mamá (...), para mí no tiene el significado del matrimonio."

Dado que no lo usa en la mano izquierda ni en el dedo anular, el anillo pasa inadvertido para las demás personas. No es frecuente que le pregunten.

Se sentiría muy mal.

Lo guardaría en un lugar seguro y no lo vendería.

"No hay anillo de matrimonio perfecto para mí que no sea este, porque me gusta mucho la plata, en relación al oro; no me gusta que sea tan ostentoso, tan llamativo. Me gustan las cosas sencillas y elegantes, para mí la plata lo es. No tengo algún problema en cuanto al uso del oro, alergia o esas cosas; sin embargo es por el solo gusto del color plateado sobre el dorado; incluso me han regalado unos aretes de oro y sí los uso pero me gusta mucho más la plata por elegante y sencilla." 


\section{Caso 3}

\section{Características}

del objeto

\section{¿Cómo y cuándo llegó} este anillo a tu vida?

\section{¿En qué situación}

lo usas?
Mujer con 38 años de edad, heterosexual, agnóstica, divorciada, funcionaria pública.

Argolla de plata, $8 \mathrm{~mm}$ de ancho, sin inscripciones u ornamentos, con bordes sin rebajar.

Hace tres años, una de sus dos amigas más cercanas tuvo la idea de mandar hacer argollas para cada una.

Uso la argolla cuando voy a salir con ellas (las amigas, a las que considera sus esposas), con las tres o con una de ellas y también uso la argolla cuando viajo, o cuando tengo actividades de trabajo, porque trabajo en un ambiente muy masculino donde las mujeres de mi edad usualmente no son solteras, como no me gustan las preguntas, ni el asedio, lo uso sobre todo cuando voy a viajar para que nadie me hable, para sentirme protegida, para que no hagan preguntas y de hecho me ha salvado de varias, porque por el tipo de ambiente de trabajo no solo es muy masculino sino muy jerárquico, entonces es muy común que los varones heterosexuales se sientan con el poder suficiente y, por lo tanto, con derecho de tener acercamientos que para mi gusto son inapropiados, entonces cuando ven que las mujeres son solteras, aparentemente están disponibles, de repente se sienten muy autorizados para tener acercamientos inadecuados y yo con eso como que los bloqueo. Me sirve como una barrera de protección."

Según el contexto, el significado del anillo se modifica. Con sus amigas significa su amistad, en situaciones profesionales significa compromiso, exclusividad sexual y emocional, falta de disponibilidad.

Para sus amigas refiere a su relación. Para las demás personas significa compromiso.

Mandaría hacer otro igual y no informaría a sus amigas sobre el extravío.

Lo guardaría en un lugar seguro.

"El anillo perfecto de matrimonio no sería de plata, seguro que no, sería de oro blanco y no sería tosco y sencillo como este, sería bastante más estilizado y seguramente no me lo habría dado una de mis amigas, me lo habría dado un tipo con el que quiera estar el resto de mi vida, en un ritual formal." 


\section{Caso 4}

\section{Características del objeto}

\section{¿Cómo y cuándo llegó} este anillo a tu vida?

\section{¿En qué situación lo usas?}

\section{¿Qué significa este anillo para ti?}

¿Qué crees que significa este anillo para las demás personas?

¿Qué pasaría si este anillo se extraviara? ¿Cómo te sentirías? ¿Qué harías?

Si en algún momento de tu vida, tuvieras que dejar de usarlo, ¿Qué harías con él?

Si pudieras tener el anillo perfecto, ¿cómo sería? ¿en qué circunstancias habría llegado a tu vida?
Mujer con 33 años de edad, heterosexual, católica, casada, madre de un hijo, arquitecta.

Anillo de oro liso, con inscripción de fecha y nombre del cónyuge en el interior, con bordes rebajados.

Matrimonio, hace cinco años.

Sólo en ocasiones especiales, ya que no está habituada a utilizar objetos en las manos.

"(...) creo en una significación muy fuerte respecto de ese anillo, que significa el amor, la fidelidad, la unión por siempre, de hecho le decía a mi esposo que a no me hubiera importado casarme solo por la Iglesia, me hubiera bastado con las palabras que nos dijimos en el altar (...), es un promesa que haces ante Dios y ante él por más que te divorcies."

"Para las demás personas, a mi parecer les es incómodo el uso del anillo de matrimonio, porque no pueden desarrollarse con mucha libertad, porque les hacen burla de que ya eres casado; sin embargo creo que para otras también es algo que es muy significativo, aunque esto obedecerá a la personalidad de cada quien."

"Si mi anillo de matrimonio se llegara a extraviar, no tendría la misma significación si me comprara otro. Eso pasó con el de mi esposo, que lo perdió, solía quitárselo y dejarlo por ahí y ya jamás lo recuperamos. Y yo no le he dicho nada de que se compre otro o que mande a hacer otro porque no tendría el mismo significado que tuvo en su momento, pues fue precisamente ese objeto el que se bendijo."

Lo guardaría en un lugar seguro.

Mi anillo perfecto no me gustaría tan ostentoso, porque en general las cosas no me gustan así, más bien, quisiera que fuera muy simple (...). Para ser perfecto, tendría que ser similar a este pero más delgado (...); el momento perfecto, quizás en un aniversario de bodas porque en algún otro evento, no le encuentro la relación en regalar un anillo con la unión que significa." 


\section{Referencias}

Aguilar, A. (1991). Anillo grabado. On 15 Exitos norteños. México: Musart.

Ayers, T., \& Brown, P. (2012). The Essential Guide to Gay and Lesbian Weddings.

Bacchiocchi S. (2013). El origen de la alianza. Recuperado el 6 de abril de 2013, de http://www.contestandotupregunta.org/ Santuario/new2/origenalianza.htm.

Berdanier, P. F. (1939). How it Began: United Featured sundicate Incorporated.

Carcopino J. (1964). Daily llife in ancient Rome. The people and the city at the height of the Empire, Great Britain, Penguin books Ltd.

Clowes, J. (1832). The Golden Wedding Ring or Observations on the Institution of Marriage. Londres: Simpkin and Marshall.

Cooper, J. (2002). Psicología social, México: Thomson.

Robb, S. (2011). "De dónde viene la tradición de usar anillos de boda". Recuperado el 5 de mayo 2013, http://www.bbc. co.uk/mundo/noticias/2011/04/110408_boda_real_anillos_ boda_tradicion_pl.shtml.

García Gual, C. (2004). Introducción a la mitología griega, Madrid, Alianza.

Geertz, C. (2005). La interpretación de las culturas, Barcelona, Gedisa.

Grijelmo, A. (2004). La seducción de las palabras, España; Santillana

Lévi-Strauss, C. (1998), Las estructuras elementales del parentesco. Barcelona: Paidós Ibérica.

Livi, R. (1991). Par de anillos. On Par de Anillos. México:

Discos Sabinas DISA México.
Mair, L. (1971). Marriage. Harmondsworth: Penguin.

Martín, F. (2002) Contribuciones para una antropología del diseño, Barcelona: Gedisa.

Mauss, M. (1967). The Gift: Forms and functions of Exchange in Archaic Societies. Nueva York: The Norton Library.

McDaniel, C., Gates, R. H., \& Gates, R. (2005). Investigación de mercados (Thomson Ed. 6 ed.).

Merton, R. K. (1990). Focused Interview. Nueva York, Estados Unidos: The Free Press.

Ogletree, S. M. (2010). With this Ring, I Thee Wed: Relating Gender Roles and Love Styles to Attitudes towards Engagement Rings and Weddings. Gender Issues, Vol. 27 (No. 1-2), p- 67-77.

Parsons, K. (2008). Subverting the Fellowship of the Wedding Ring. Journal of Social Philosophy, 39(3), 393-410. doi:10.1111/j.1467-9833.2008.00432.x

Uller, T., \& Johansson, L. C. (2003). Human mate choice and the wedding ring effect. Human Nature, Vol. 14 (No. 3), pp. 267-276.

Wojtyła, K. J. (1960), The Jeweler's Shop. Recuperado de http://web1.desales.edu/assets/salesian/PDF/ JewelersShopscriptact1.pdf.

Howard, V. (2003). A real man's ring: Gender and the invention of tradition. Journal of Social History, Vol. 36, No. 4, p. 837-856.

Monger, G. (2004). Marriage Customs of the World: From Henna to Honeymoons. California: ABC-CLIO. 